\title{
Electron temperature fluctuations in Seyfert galaxies
}

\author{
Rogemar A. Riffel, ${ }^{1 \star}$ Oli L. Dors, ${ }^{2}$ Angela C. Krabbe, ${ }^{2}$ César Esteban ${ }^{3,4}$ \\ ${ }^{1}$ Departamento de Física, Centro de Ciências Naturais e Exatas, Universidade Federal de Santa Maria, 97105-900, Santa Maria, RS, Brazil \\ ${ }^{2}$ Universidade do Vale do Paraíba, Av. Shishima Hifumi, 2911, Cep 12244-000, São José dos Campos, SP, Brazil \\ 3 Instituto de Astrofísica de Canarias (IAC), E-38205 La Laguna, Spain \\ ${ }^{4}$ Departamento de Astrofísica, Universidad de La Laguna, E-38206 La Laguna, Spain
}

Accepted XXX. Received YYY; in original form ZZZ

\begin{abstract}
We use Gemini GMOS-IFU observations of three luminous nearby Seyfert galaxies (Mrk 79, Mrk 348 and Mrk 607) to estimate the electron temperature $\left(T_{\mathrm{e}}\right)$ fluctuations in the inner $0.4-1.1 \mathrm{kpc}$ region of these galaxies. Based on $T_{\mathrm{e}}$ determinations through the $[\mathrm{O} \mathrm{III}] \lambda 5007 / \lambda 4363$ emission line ratio of each spaxel, temperature variations are quantified by computing the integrated value of the temperature fluctuation parameter $\left(t^{2}\right)$ projected in the plane of the sky $t_{\mathrm{A}}^{2}$, for the first time in Active Galactic Nuclei. We find $t_{\mathrm{A}}^{2}$ values of $0.135,0.039$, and 0.015 for Mrk 79, Mrk 348, and Mrk607, respectively, which are of the same order or larger than the maximum values reported in star-forming regions and planetary nebulae. Taking into account that $t_{\mathrm{A}}^{2}$ should be considered a lower limit of the total $t^{2}$ in the nebular volume, the results suggest that the impact of such fluctuations on chemical abundance determinations can be important in some AGNs.
\end{abstract}

Key words: galaxies: Seyfert - galaxies: active - galaxies: abundances - galaxies: ISM

\section{INTRODUCTION}

Metal abundance determinations in the gas phase of line emitting objects, such as star-forming regions (SFs), Planetary Nebulae (PNs) and Active Galactic Nuclei (AGNs), play a key role in the studies of chemical enrichment of the interstellar medium (ISM) and of the cosmic evolution of the Universe. Therefore, along decades several efforts have been made in developing methods to derive the chemical abundance and metallicity in these objects.

There is consensus that, for SFs and PNs, reliable metal abundance determinations are those based on direct determination of the electron temperature $\left(T_{\mathrm{e}}\right)$, which is obtained through the measurements of auroral lines sensitive to $T_{\mathrm{e}}$, for instance, $[\mathrm{O}$ III $] \lambda 4363$, [N $\mathrm{NI}] \lambda 5755$ and $[\mathrm{S} \mathrm{III}] \lambda 6312$. This approach is called the $T_{\mathrm{e}}$-method (for a review see Peimbert et al. 2017; Pérez-Montero 2017) and it has been used to directly determine the chemical abundance of thousands of local SFs (e.g. Smith 1975; Torres-Peimbert et al. 1989; van Zee et al. 1998; Kennicutt et al. 2003; Hägele et al. 2008; Zurita \& Bresolin 2012; Berg et al. 2020, among others) as well as for some objects at high redshifts $(z>1$; e.g. Sanders et al. 2016, 2020; Gburek et al. 2019). There is considerable observational evidence that supports the reliability of $T_{\mathrm{e}}$-method, such as, for example, the agreement between oxygen abundances obtained for SFs located in the solar neighborhood and those obtained from observations of the weak interstellar O I $\lambda 1356$ line towards stars (see Pilyugin 2003 and references

* E-mail: rogemar@ufsm.br therein), as well as between oxygen abundances obtained for SFs and B-type stars in the Milky Way and other nearby galaxies (see Toribio San Cipriano et al. 2017 and references therein).

However, abundance determinations using the $T_{\mathrm{e}}$-method are subject to some caveats. It is worth to state from the onset that, Peimbert (1967) proposed the presence of electron temperature fluctuations in $\mathrm{H}$ II regions. In that work, temperatures based on collisionally excited lines (CELs), i.e. $[\mathrm{O}$ III $](\lambda 4949+\lambda 5007) / \lambda 4363$, from the SFs M 8, M 17 and Orion Nebulae were found to be $2000-4000 \mathrm{~K}$ higher than those determined from the Balmer continuum and radio observations (see also García-Rojas \& Esteban 2007). Thereafter, Peimbert \& Costero (1969) reported that temperature variations over the observed volume of the nebula, if not taken into account, produce underestimated gaseous abundances derived through intensity ratios of CELs using the $T_{\mathrm{e}}$-method.

In fact, carbon and oxygen abundances derived from faint optical recombination lines (ORLs) (almost independent from $T_{\mathrm{e}}$ ) of SFs are higher by a factor of $0.2-0.4$ dex than those derived from the $T_{\mathrm{e}}$-method (e.g. Tsamis et al. 2003; Esteban et al. 2004; Peimbert et al. 2005; García-Rojas et al. 2006). This is known as the abundance discrepancy problem and several scenarios have been proposed to explain it (for a review see García-Rojas \& Esteban 2007). If this discrepancy is due to presence of electron temperature fluctuations (quantified by the $t^{2}$ parameter), as suggested by Peimbert \& Costero (1969), large variations of $T_{\mathrm{e}}$ along gaseous nebulae could be derived to a level of $t^{2} \sim 0.04$ (e.g. García- 
Rojas \& Esteban 2007). However, observations of SFs over decades have failed to find direct evidence for such high levels of electron temperature fluctuations. A discrete estimation of $t^{2}$ in the plane of the sky, $t_{\mathrm{A}}^{2}$, can be obtained when one has point-to-point determinations of $T_{\mathrm{e}}$ of a given nebula. Strictly speaking, $t_{\mathrm{A}}^{2}$ should be considered a lower limit to $t^{2}$ because this last parameter, by definition, is integrated over the volume of the considered region (see further argumentation given by Rubin et al. 2003 and Mesa-Delgado et al. 2008). Using this approximation and temperature measurements based on the $[\mathrm{O}$ III $](\lambda 4959+\lambda 5007) / \lambda 4363$ line ratio, Krabbe \& Copetti (2002) found $t_{\mathrm{A}}^{2} \sim 0.0025$, or equivalently a temperature dispersion of only $5 \%$ in the 30 Doradus Nebula. Other estimates of $t_{\mathrm{A}}^{2}$ for extragalactic SFs have derived similar low values of this parameter (Tsamis et al. 2003; Oliveira et al. 2008). Rubin et al. (2003), by using Hubble Space Telescope (HST/STIS) long-slit spectroscopy of the Orion Nebula, found $t_{\mathrm{A}}^{2}$ varying from $\sim 0.006$ to $\sim 0.018$. Recent radio observations in different zones of the Orion Nebula by Wilson et al. (2015) revealed only spatial variations in $T_{\mathrm{e}}$ of the order of $13 \%$. Moreover, a low $t^{2}$ value of $\sim 0.005$ was found from photoionization model simulations for SFs by Copetti (2006).

Hitherto, the $T_{\mathrm{e}}$-method has been used to estimate the elemental abundances of oxygen and nitrogen for few AGNs (see Alloin et al. 1992; Izotov \& Thuan 2008; Dors et al. 2015, 2017; Flury \& Moran 2020; Dors et al. 2020b), which are mostly based on photoionization models (see Dors et al. 2020a and references therein). Gaseous abundance determinations in AGNs through ORLs are not available in the literature, mainly because these lines are extremely faint and are only accessible in nearby objects. In particular, Dors et al. (2020b) showed that $\mathrm{O} / \mathrm{H}$ estimates based on $T_{\mathrm{e}}$-method in the Narrow Line Regions (NLRs) of AGNs are underestimated by an average factor of about 0.2 dex in comparison with those derived from detailed photoionization models, where the discrepancy is more pronounced (reaching $\sim 0.8 \mathrm{dex}$ ) for the low metallicity regime $\left[12+\log (\mathrm{O} / \mathrm{H}) \lesssim 8.4\right.$ or $\left.\left(Z / \mathrm{Z}_{\odot}\right) \lesssim 0.5\right]$.

In contrast to SFs, only a few studies have addressed the electron temperature structure in AGNs. For example, Revalski et al. (2018a,b) found no systematic electron temperature variation along the radius of three nearby AGNs using long slit spectroscopy. On the other hand, electron temperature maps for the inner kpc of luminous Seyfert galaxies, derived using the $[\mathrm{O}$ III $](\lambda 4959+\lambda 5007) / \lambda 4363$ line ratio measured from integral field spectroscopy, show values varying from $\sim 8000$ to $\gtrsim 30000 \mathrm{~K}$ (Riffel et al. 2021). These findings demonstrate - for the first time - that large spatial variations of $T_{\mathrm{e}}$ are present in AGNs, providing a unique opportunity to explore the effects of $t^{2}$ on abundance determinations in this object class, which is a benchmark to study the properties of distant galaxies and the high-redshift Universe.

Although temperature fluctuations have been quantified for SFs and PNs, this is the first study aimed at studying such fluctuations in AGNs. We present a study on the electron temperature fluctuations in three Seyfert galaxies (Mrk 79, Mrk 348 and Mrk 607) using 2D spectroscopy data presented by Riffel et al. (2021). The data present high-spatial and spectral resolution spectra and will provide detailed information about the spatial distribution of the physical properties of these galaxies. In Section 2, we describe the data and methodology, in Sect. 3 we present our results and Sect. 4 is aimed to evaluate the electron temperature fluctuations. Finally, the conclusions are presented in Sect. 5 .

\section{OBSERVATIONAL DATA}

We use optical emission-line flux distributions to map the electron density and electron temperature in the $0.4-1.1 \mathrm{kpc}$ inner central region of Mrk 79, Mrk 348 and Mrk607, three luminous nearby Seyfert galaxies. These objects were selected from Freitas et al. (2018) by presenting extended [O III] $\lambda 4363$ emission (see Riffel et al. 2021).

The data were obtained with the Gemini Multi-Object Spectrograph (GMOS, Allington-Smith et al. 2002) Integral Field Unit (IFU), covering the spectral range from 4300 to $7100 \AA$ with a velocity resolution of $\sim 90 \mathrm{~km} \mathrm{~s}^{-1}$ (estimated by measuring the full width at half-maximum, FWHM, of emission-line profiles of the CuAr calibration lamp, used to wavelength calibrate the data) and spatial resolutions of 110-280 pc (estimated as the FWHM of the continuum flux distribution of field stars from the acquisition images). The data reduction procedure is described in Freitas et al. (2018) whereby the standard procedures using the GEMINI.IRAF package were followed. Here, we use the emissionline flux distributions measured by Riffel et al. (2021), who used the IFSCUBE python package (Ruschel-Dutra 2020) to investigate the variations of the electron temperature in the NLRs of the three Seyfert galaxies. Table 1 presents the basic information of the three galaxies.

We recompute the electron temperatures using the PyNeb routine (Luridiana et al. 2015) and the $\frac{I\left(\left[\mathrm{O}_{\mathrm{III}}\right] \lambda 5007\right)}{I\left(\left[\mathrm{O}_{\mathrm{III}}\right] \lambda 4363\right)}$ and $\frac{I([\mathrm{~S} I]] \lambda 6716)}{I([\mathrm{~S} \text { II }] \lambda 6731)}$ emission-line intensity ratios from Riffel et al. (2021). For the highest temperatures $\left(T_{\mathrm{e}} \gtrsim 30000 \mathrm{~K}\right)$, the PyNeb based measurements are expected to be more accurate than those based on the equation from Hägele et al. (2008) assumed in Riffel et al. (2021), as the range of temperatures used to derive this equation originally does not include high temperatures. First, we compute the electron density $\left(N_{\mathrm{e}}\right)$ values for each spaxel using the [S II] line ratio and assuming $T_{\mathrm{e}}=20000 \mathrm{~K}$; then we calculate the electron temperature assuming the $N_{e}$ values obtained for each spaxel. Although the [S II] emission arises from low ionization degree and partiallyionized zones and the [O III] emission traces fully ionized and perhaps higher density zones, the dependence of $T_{\mathrm{e}}$ on the adopted $N_{e}$ appears to be negligible.

\section{RESULTS}

The electron density maps for Mrk 79, Mrk 348 and Mrk 607 were already shown in Riffel et al. (2021). The $N_{\mathrm{e}}$ values range from $\sim 100 \mathrm{~cm}^{-3}$ to $\sim 2300 \mathrm{~cm}^{-3}$. These values are in the range of densities reported for the central region of nearby active galaxies (e.g. Kakkad et al. 2018; Freitas et al. 2018). The electron temperature maps for Mrk 79, Mrk 348 and Mrk 607 are shown in Fig. 1. These maps are consistent with those presented in Riffel et al. (2021) based on the equation from Hägele et al. (2008), but as pointed out in Sec. 2, the PyNeb based values are more accurate for the highest temperature values. The $T_{e}$ values in these galaxies are notably higher than those found in SFs (e.g. Kennicutt et al. 2003; Hägele et al. 2008) and in PNs (e.g. Krabbe \& Copetti 2005; Sandin 

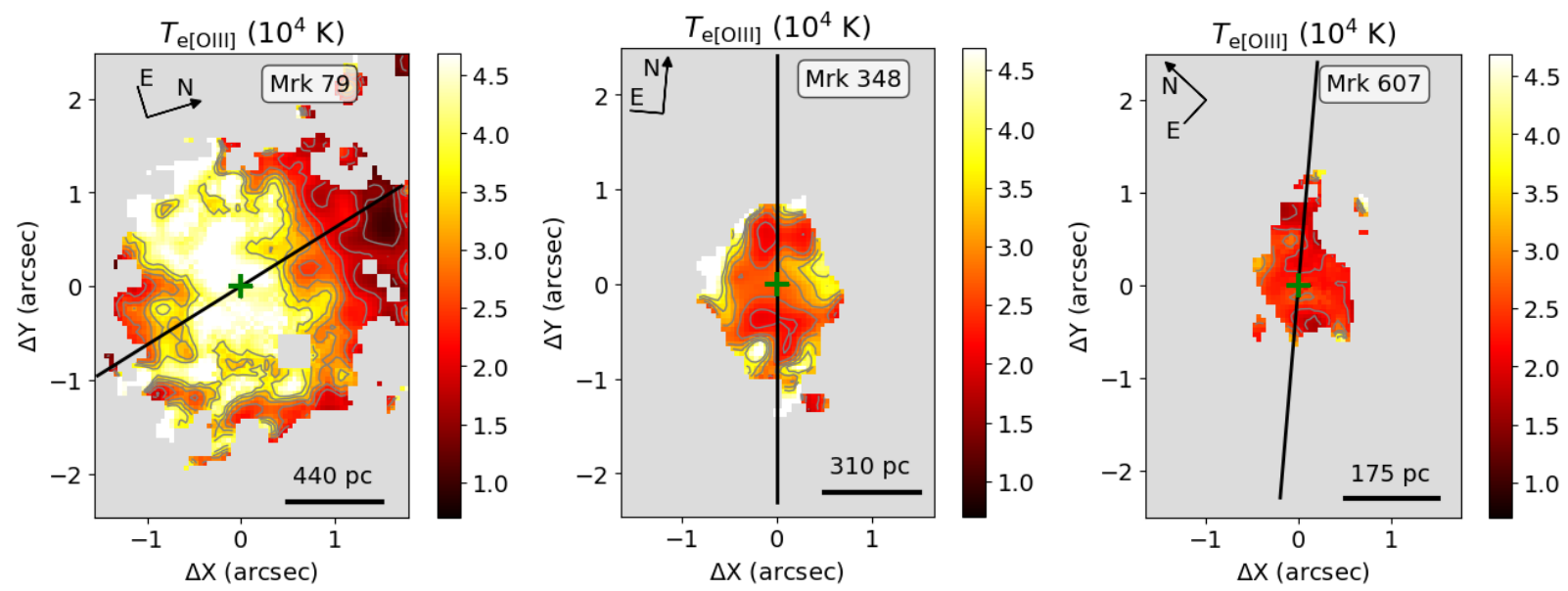

Figure 1. Maps of the electron temperature for Mrk 79 (left), Mrk 348 (middle) and Mrk 607 (right). The central crosses mark the position of the galaxy nuclei and the lines show the orientation of the AGN ionization axes, as obtained from [O III] HST images by Schmitt et al. (2003). The physical scale and the spatial orientation of the GMOS field of view for each object are shown. The gray regions correspond to locations where the $[\mathrm{O} \mathrm{III}] 4363$ emission line is not detected at a $3 \sigma$ continuum level.
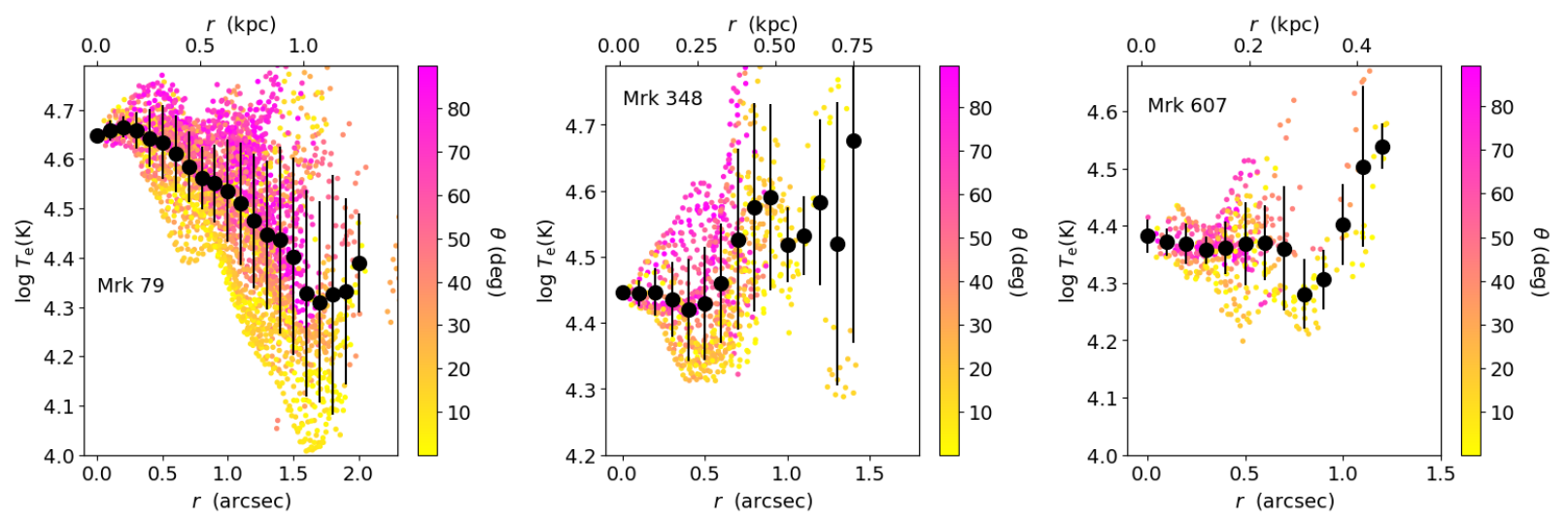

Figure 2. Plots of the logarithm of $T_{\mathrm{e}}$ versus the distance (in units of arcsec) from the continuum peak for Mrk 79 (left), Mrk 348 (middle) and Mrk 607 (right). The points are color coded in according to the angle $(\theta)$ between the position of each spaxel and the orientation of the AGN ionization axis (indicated in Fig. 1), whose values are shown in degrees in the color bar. The filled circles represent the mean $T_{\mathrm{e}}$ values and the error bars the standard deviation of these within bins of 0.1 arcsec.
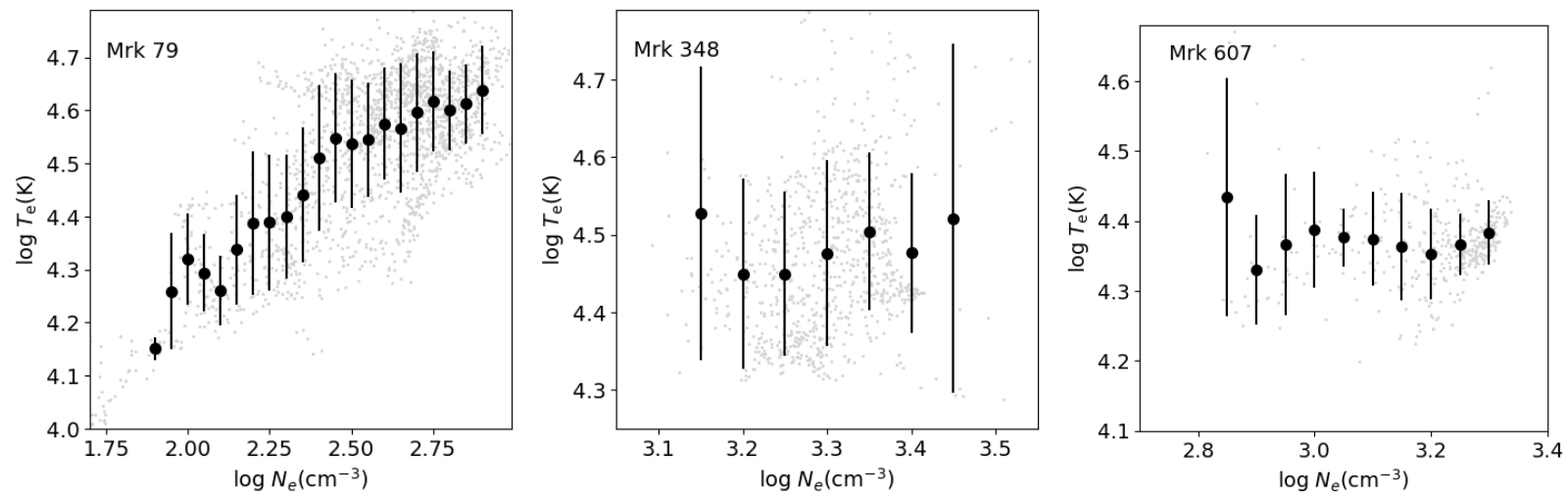

Figure 3. Plots of the logarithm of $T_{\mathrm{e}}$ versus the logarithm of $N_{\mathrm{e}}$ for Mrk 79 (left), Mrk 348 (middle) and Mrk 607 (right). The gray points show the values of all spaxels and the filled circles show the mean temperature values within bins of $\log N_{\mathrm{e}} /\left(\mathrm{cm}^{-3}\right)=0.05$. The error bars are the standard deviation of the $T_{\mathrm{e}}$ values within each bin. 
Table 1. Basic information of Mrk 79, Mrk 348 and Mrk607. Columns: (1) Name of the galaxy, (2) redshift (z), (3) nuclear activity class, (4) bolometric luminosity $\left(L_{\mathrm{bol}}\right)$ calculated using $\log L_{\mathrm{bol}}=0.0378\left(\log L_{\mathrm{X}}\right)^{2}-2.03 \log L_{\mathrm{X}}+61.6$ (Ichikawa et al. 2017), where $L_{\mathrm{X}}$ is the hard X-ray (measured in the range 14-195 keV) luminosity from Oh et al. (2018), (5) exposure time, (6) spatial resolution estimated as the FWHM of the continuum flux distribution of field stars from the GMOS acquisition images (see Freitas et al. 2018).

\begin{tabular}{lccccc}
\hline Object & $z$ & Nuc. Act. & $\begin{array}{c}\log L_{\text {bol }} \\
\left(\mathrm{erg} \mathrm{s}^{-1}\right)\end{array}$ & $\begin{array}{c}\text { Exp. Time } \\
(\mathrm{sec})\end{array}$ & $\begin{array}{c}\text { Spat. Res. } \\
(\mathrm{pc})\end{array}$ \\
\hline Mrk 79 & 0.0222 & Sy 1 & 45.0 & $6 \times 810$ & $280 \pm 30$ \\
Mrk 348 & 0.0150 & Sy 2 & 45.3 & $6 \times 810$ & $190 \pm 25$ \\
Mrk 607 & 0.0089 & Sy 2 & 43.4 & $7 \times 810$ & $110 \pm 14$ \\
\hline
\end{tabular}

et al. 2008; Rauber et al. 2014; Monreal-Ibero \& Walsh 2020), which are in the order of $10000-15000 \mathrm{~K}$. The highest $T_{\mathrm{e}}$ values are derived in regions away from the AGN ionization axis in all galaxies. This behaviour is clearly seen in Fig. 2, a plot of $T_{\mathrm{e}}$ versus the distance to the nucleus, where the points are color coded in according to the angle $(\theta)$ between the position of each spaxel and the orientation of the AGN ionization axis (indicated in Fig. 1). The $T_{\mathrm{e}}$ values along the AGN ionization axis are similar to those obtained using long slit spectroscopy and predicted by photoionization models, i.e. in the range $\sim 10000-25000 \mathrm{~K}$ (Revalski et al. 2018a,b, 2021). However, the highest $T_{\mathrm{e}}$ values $(\gtrsim 30000 \mathrm{~K}$ ) are derived outside the AGN ionization axis, originated probably by shock ionization rather than photoionization (Riffel et al. 2021). The wide range of temperature variations derived in the galaxies studied here is consistent with the [O III $] \lambda 4363 /[\mathrm{O}$ III $] \lambda 5007$ map for NGC 1068 shown by D'Agostino et al. (2019), which presents values in the range $0.02-0.08$, corresponding to temperatures of $\sim 15000-40000 \mathrm{~K}$, as obtained using the PyNeb routine and assuming $N_{\mathrm{e}}=1000 \mathrm{~cm}^{-3}$. Our results indicate the presence of large spatial variations of electron temperature in the gas phase of AGNs, not observed in SFs and $\mathrm{PNS}$.

In Fig. 3 we show plots of $T_{\mathrm{e}}$ versus $N_{\mathrm{e}}$ for Mrk 79 (left), Mrk 348 (middle) and Mrk 607 (right). The filled circles show the $T_{\mathrm{e}}$ values within bins of $\log N_{\mathrm{e}} /\left(\mathrm{cm}^{-3}\right)=0.05$. A clear correlation between $T_{\mathrm{e}}$ and $N_{\mathrm{e}}$ is observed for Mrk 79, while no correlations are seen for Mrk 348 and Mrk 607. Similar correlations are found in ring-shaped PNs by Krabbe \& Copetti (2005). A possible interpretation for the correlation seen in Mrk 79 is that the lower $T_{\mathrm{e}}$ and $N_{\mathrm{e}}$, seen along the AGN ionization axis, are mainly due to photoionization by the AGN radiation, while the increase of the values of these properties with the distance to the ionization axis indicates a larger contribution of emission of compressed shock-ionized gas.

\section{ELECTRON TEMPERATURE FLUCTUATIONS AND IMPLICATIONS}

According to Peimbert \& Costero (1969), the temperature fluctuations over the observed gas phase volume $(V)$ of a nebula can be quantified in terms of the mean electron temperature $\left(T_{0}\right)$ and the $t^{2}$ parameter, which are defined as

$T_{0}=\frac{\int T_{\mathrm{e}} N_{\mathrm{e}} N_{\mathrm{i}} \mathrm{d} V}{\int N_{\mathrm{e}} N_{\mathrm{i}} \mathrm{d} V}$

and

$t^{2}=\frac{\int\left(T_{\mathrm{e}}-T_{0}\right)^{2} N_{i} N_{\mathrm{e}} \mathrm{d} V}{T_{0}^{2} \int N_{i} N_{\mathrm{e}} \mathrm{d} V}$, where $N_{i}$ is the density of the ion used to measure the temperature. A direct estimate of $t^{2}$ along the line-of-sight can not be obtained from our data, but we can estimate $t^{2}$ projected in the plane of the sky $\left(t_{\mathrm{A}}^{2}\right)$ - as well as its associated $T_{0, \mathrm{~A}}-$ using the equations given by:

$T_{0, \mathrm{~A}}=\frac{\sum_{j} T_{\mathrm{e}, j} / T_{\mathrm{e}, j}^{\mathrm{err}}}{\sum_{j} 1 / T_{\mathrm{e}, j}^{\mathrm{err}}}$

and

$t_{\mathrm{A}}^{2}=\frac{\sum_{j}\left(T_{\mathrm{e}, j}-T_{0, A}\right)^{2} / T_{\mathrm{e}, j}^{\mathrm{err}}}{T_{0, A}^{2} \sum_{j} 1 / T_{\mathrm{e}, j}^{\mathrm{err}}}$,

where $T_{\mathrm{e}, j}$ and $T_{\mathrm{e}, j}^{\mathrm{err}}$ are the electron temperature and its uncertainty in each spaxel, respectively. We use $1 / T_{\mathrm{e}, j}^{\mathrm{err}}$ as a weighting factor of the point-to-point temperature instead of $N_{i} N_{\mathrm{e}}$ (as in Rubin et al. 2003 or Mesa-Delgado et al. 2008) for various reasons. Firstly, because $N_{\mathrm{e}}$ is determined from a [S II] line ratio, and this indicator may not be representing well the density - and density variations - within the $\mathrm{O}^{++}$ zone in a huge and complex object as an AGN host. Secondly, as it has been seen in Sect. 3, the spaxels with higher $T_{\mathrm{e}}$ values - and with a larger contribution of shock excitation tend to be located in the regions away from the AGN ionization axis in all galaxies, and these correspond to the faintest zones. Since the emissivity of recombination or collisionally excited lines of typical ionized nebulae are proportional to the $N_{i} N_{\mathrm{e}}$ factor, lines affected by shock excitation are very much dependent on shock velocity and other geometrical and physical aspects of the interaction. The factor $1 / T_{\mathrm{e}, j}^{\mathrm{err}}$ is basically proportional to the signal-to-noise ratio of the auroral [O III] $\lambda 4363$ line, therefore, in this case, we think it is a better choice for weighting the contribution of each spaxel to define $t_{\mathrm{A}}^{2}$.

The $T_{0, \mathrm{~A}}$ and $t_{\mathrm{A}}^{2}$ values derived for the three galaxies are listed in the third and fourth columns of Table 2. These parameters are calculated assuming the full area with [O III $] \lambda 4363$ line detected above a $3 \sigma$ level. The electron temperature calculated by summing up the fluxes of all spaxels where the $[\mathrm{O}$ III $] \lambda 4363$ is detected $-T_{\mathrm{e}, \text { sum }}-$ is also included in the second column of Table 2. These quantities may be considered as representative of each entire object. The $t_{\mathrm{A}}^{2}$ values we find are $0.135,0.039$, and 0.015 for Mrk 79, Mrk 348, and Mrk607, respectively, implying spatial variations of $T_{e}$ between 12 and $37 \%$. The $t_{\mathrm{A}}^{2}$ found in the AGNs studied here are of the order or even much higher (up to a factor about 7 ) than the maximum values derived in observational studies of extragalactic SFs (e.g. Krabbe \& Copetti 2002; Oliveira et al. 2008), the Orion Nebula (e.g. Rubin et al. 2003; MesaDelgado et al. 2008) or PNe (e.g. Krabbe \& Copetti 2005; 
Table 2. Electron temperature $\left(T_{\mathrm{e}, \mathrm{sum}}\right)$ calculated by summing up the fluxes from the spaxels with $T_{e}$ measurements, mean electron temperature $\left(T_{0, A}\right)$, and surface temperature fluctuation $\left(t_{\mathrm{A}}^{2}\right)$ for the three objects. The uncertainties in the temperatures results from propagating the $1 \sigma$ error of the $[\mathrm{O}$ III $] \lambda 5007 / \lambda 4363$ flux line ratio.

\begin{tabular}{lccc}
\hline Object & $\begin{array}{c}T_{\mathrm{e}, \text { sum }} \\
\left(10^{4} \mathrm{~K}\right)\end{array}$ & $\begin{array}{c}T_{0, \mathrm{~A}} \\
\left(10^{4} \mathrm{~K}\right)\end{array}$ & $t_{\mathrm{A}}^{2}$ \\
\hline Mrk 79 & $3.74_{-0.35}^{+0.49}$ & $3.00_{-0.37}^{+0.59}$ & 0.135 \\
Mrk 348 & $2.88_{-0.29}^{+0.43}$ & $2.79_{-0.38}^{+0.37}$ & 0.039 \\
Mrk 607 & $2.40_{-0.17}^{+0.21}$ & $2.36_{-0.24}^{+0.34}$ & 0.015 \\
\hline
\end{tabular}

Rauber et al. 2014). Our results suggest that the potential impact of $t^{2}$ on abundance determinations based on the $T_{\mathrm{e}^{-}}$ method may be important for some AGN hosts.

\section{CONCLUSIONS}

We have studied the temperature fluctuations in the inner 0.4-1.1 kpc region of three nearby Seyfert galaxies: Mrk 79, Mrk 348, and Mrk 607. In particular, for Mrk 79, an AGN with strong ionized outflows, we derive a direct relation between electron temperature and electron density, possibly caused by compressed shock-ionized gas. The temperature fluctuations are quantified by the $t_{\mathrm{A}}^{2}$ parameter, which is derived in the range of $0.015-0.135$, of the order or even larger than the maximum values obtained for galactic and extragalactic star forming regions (H II regions) and for planetary nebulae. Therefore, the effect of temperature fluctuations may be important in gas phase abundance estimates in AGNs based on $T_{\mathrm{e}}$-method.

\section{ACKNOWLEDGEMENTS}

We thank an anonymous referee for their critical reading and suggestions that helped us to improve our paper. R.A.R. acknowledges support from Conselho Nacional de Desenvolvimento Científico e Tecnológico (CNPq) and Fundação de Amparo à Pesquisa do Estado do Rio Grande do Sul (FAPERGS). O.L.D. and A.K. acknowledge support from $\mathrm{CNPq}$ and Fundação de Amparo à Pesquisa do Estado de São Paulo (FAPESP). C.E. acknowledges support from the Agencia Estatal de Investigación del Ministerio de Ciencia e Innovación (AEI-MCINN) under grant Espectroscopía de campo integral de regiones HII locales. Modelos para el estudio de regiones HII extragalácticas with reference 10.13039/501100011033. Based on observations obtained at the Gemini Observatory, which is operated by the Association of Universities for Research in Astronomy, Inc., under a cooperative agreement with the NSF on behalf of the Gemini partnership: the National Science Foundation (United States), National Research Council (Canada), CONICYT (Chile), Ministerio de Ciencia, Tecnología e Innovación Productiva (Argentina), Ministério da Ciência, Tecnologia e Inovação (Brazil), and Korea Astronomy and Space Science Institute (Republic of Korea).

\section{DATA AVAILABILITY}

The data used in this paper is available in the Gemini Science Archive under the project code GN-2014B-Q-87.

\section{REFERENCES}

Allington-Smith J., et al., 2002, PASP, 114, 892

Alloin D., Bica E., Bonatto C., Prugniel P., 1992, A\&A, 266, 117

Berg D. A., Pogge R. W., Skillman E. D., Croxall K. V., Moustakas J., Rogers N. S. J., Sun J., 2020, ApJ, 893, 96

Copetti M. V. F., 2006, A\&A, 453, 943

D'Agostino J. J., et al., 2019, MNRAS, 487, 4153

Dors O. L., Cardaci M. V., Hägele G. F., Rodrigues I., Grebel E. K., Pilyugin L. S., Freitas-Lemes P., Krabbe A. C., 2015, MNRAS, 453, 4102

Dors O. L. J., Arellano-Córdova K. Z., Cardaci M. V., Hägele G. F., 2017, MNRAS, 468, L113

Dors O. L., et al., 2020a, MNRAS, 492, 468

Dors O. L., Maiolino R., Cardaci M. V., Hägele G. F., Krabbe A. C., Pérez-Montero E., Armah M., 2020b, MNRAS, 496, 3209

Esteban C., Peimbert M., García-Rojas J., Ruiz M. T., Peimbert A., Rodríguez M., 2004, MNRAS, 355, 229

Flury S. R., Moran E. C., 2020, MNRAS, 496, 2191

Freitas I. C., et al., 2018, MNRAS, 476, 2760

García-Rojas J., Esteban C., 2007, ApJ, 670, 457

García-Rojas J., Esteban C., Peimbert M., Costado M. T., Rodríguez M., Peimbert A., Ruiz M. T., 2006, MNRAS, 368, 253

Gburek T., et al., 2019, ApJ, 887, 168

Hägele G. F., Díaz Á. I., Terlevich E., Terlevich R., Pérez-Montero E., Cardaci M. V., 2008, MNRAS, 383, 209

Ichikawa K., Ricci C., Ueda Y., Matsuoka K., Toba Y., Kawamuro T., Trakhtenbrot B., Koss M. J., 2017, ApJ, 835, 74

Izotov Y. I., Thuan T. X., 2008, ApJ, 687, 133

Kakkad D., et al., 2018, A\&A, 618, A6

Kennicutt Robert C. J., Bresolin F., Garnett D. R., 2003, ApJ, 591,801

Krabbe A. C., Copetti M. V. F., 2002, A\&A, 387, 295

Krabbe A. C., Copetti M. V. F., 2005, A\&A, 443, 981

Luridiana V., Morisset C., Shaw R. A., 2015, A\&A, 573, A42

Mesa-Delgado A., Esteban C., García-Rojas J., 2008, ApJ, 675, 389

Monreal-Ibero A., Walsh J. R., 2020, A\&A, 634, A47

Oh K., et al., 2018, ApJS, 235, 4

Oliveira V. A., Copetti M. V. F., Krabbe A. C., 2008, A\&A, 492, 463

Peimbert M., 1967, ApJ, 150, 825

Peimbert M., Costero R., 1969, Boletin de los Observatorios Tonantzintla y Tacubaya, 5, 3

Peimbert A., Peimbert M., Ruiz M. T., 2005, ApJ, 634, 1056

Peimbert M., Peimbert A., Delgado-Inglada G., 2017, PASP, 129, 082001

Pérez-Montero E., 2017, PASP, 129, 043001

Pilyugin L. S., 2003, A\&A, 399, 1003

Rauber A. B., Copetti M. V. F., Krabbe A. C., 2014, A\&A, 563, A42

Revalski M., Crenshaw D. M., Kraemer S. B., Fischer T. C., Schmitt H. R., Machuca C., 2018a, ApJ, 856, 46

Revalski M., et al., 2018b, ApJ, 867, 88

Revalski M., et al., 2021, arXiv e-prints, p. arXiv:2101.06270

Riffel R. A., et al., 2021, MNRAS, 501, L54

Rubin R. H., Martin P. G., Dufour R. J., Ferland G. J., Blagrave K. P. M., Liu X. W., Nguyen J. F., Baldwin J. A., 2003, MNRAS, 340, 362

Ruschel-Dutra D., 2020, danielrd6/ifscube v1.0, doi:10.5281/zenodo.3945237, https://doi.org/10.5281/ zenodo. 3945237 
Sanders R. L., et al., 2016, ApJ, 825, L23

Sanders R. L., et al., 2020, MNRAS, 491, 1427

Sandin C., Schönberner D., Roth M. M., Steffen M., Böhm P., Monreal-Ibero A., 2008, A\&A, 486, 545

Schmitt H. R., Donley J. L., Antonucci R. R. J., Hutchings J. B., Kinney A. L., 2003, ApJS, 148, 327

Smith H. E., 1975, ApJ, 199, 591

Toribio San Cipriano L., Domínguez-Guzmán G., Esteban C., García-Rojas J., Mesa-Delgado A., Bresolin F., Rodríguez M., Simón-Díaz S., 2017, MNRAS, 467, 3759

Torres-Peimbert S., Peimbert M., Fierro J., 1989, ApJ, 345, 186

Tsamis Y. G., Barlow M. J., Liu X. W., Danziger I. J., Storey P. J., 2003, MNRAS, 338, 687

Wilson T. L., Bania T. M., Balser D. S., 2015, ApJ, 812, 45

Zurita A., Bresolin F., 2012, MNRAS, 427, 1463

van Zee L., Salzer J. J., Haynes M. P., O’Donoghue A. A., Balonek T. J., 1998, AJ, 116, 2805

This paper has been typeset from a $\mathrm{T}_{\mathrm{E}} \mathrm{X} / \mathrm{LAT}_{\mathrm{E}} \mathrm{X}$ file prepared by the author. 